\title{
Splenic Irradiation as a Component of a Reduced-Intensity Conditioning Regimen for Hematopoietic Stem Cell Transplantation in Myelofibrosis with Massive Splenomegaly
}

\author{
Takeshi Ito, ${ }^{1}$ Kentaro Akagi, ${ }^{1}$ Tadakazu Kondo, ${ }^{1}$ Hiroshi Kawabata, ${ }^{1}$ \\ Tatsuo Ichinohe ${ }^{1}$ and Akifumi Takaori-Kondo ${ }^{1}$ \\ ${ }^{1}$ Department of Hematology and Oncology, Graduate School of Medicine, Kyoto University, Kyoto, Japan
}

\begin{abstract}
Primary myelofibrosis is a hematologic neoplasm characterized by bone marrow fibrosis and extramedullary hematopoiesis. A similar clinical condition can occur at late stage of myeloproliferative neoplasms such as polycythemia vera and essential thrombocythemia. Although allogeneic hematopoietic stem cell transplantation (HSCT) is currently the only curative strategy for both conditions, massive splenomegaly frequently observed in patients with myelofibrosis is considered to be a risk factor for graft failure or engraftment delay after transplantation. A proportion of patients can benefit from splenectomy before transplantation but such procedures have been associated with substantial surgical morbidity. Here, we report two elderly patients with myelofibrosis who received scheduled splenic irradiation for massive splenomegaly immediately prior to allogeneic HSCT instead of undergoing splenectomy. The first patient was a 60-year-old woman who received peripheral blood stem cell transplantation for post-essential thrombocythemia myelofibrosis from an HLA-identical sibling; the second patient was a 60 -year-old man who received unrelated bone marrow transplantation for primary myelofibrosis. After receiving fractionated splenic irradiation and fludarabine-based reduced-intensity conditioning regimens, these patients showed remarkable reduction of their splenomegaly at the time of transplantation. They attained successful donor cell engraftment without severe complications related to splenic irradiation, while improvement in splenomegaly was durable. Our experience suggests that splenic irradiation before allogeneic HSCT might be a safe and effective alternative to splenectomy for myelofibrosis patients with massive splenomegaly in terms of reducing the risk of surgical morbidity.
\end{abstract}

Keywords: allogeneic hematopoietic stem cell transplantation; myelofibrosis; myeloproliferative neoplasms; reducedintensity conditioning regimen; splenic irradiation

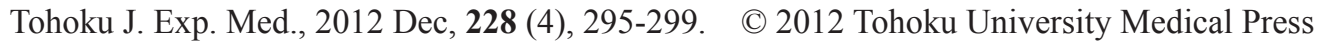

Primary myelofibrosis is a clonal hematologic neoplasm characterized by excessive proliferation of megakaryocytes and granulocytes, bone marrow fibrosis, and extramedullary hematopoiesis. Clinical manifestations include leukoerythroblastic anemia, splenomegaly, and constitutional symptoms, such as fatigue, weight loss, night sweat, and fever. Myelofibrosis can also occur at an advanced stage of other hematologic neoplasms, especially during the course of polycythemia vera and essential thrombocythemia. The median survival time is about six years with wide variation (Cervantes et al. 2009). A similar clinical condition can occur at late stage of polycythemia vera and essential thrombocythemia and also is associated with a poor prognosis.

Allogeneic hematopoietic stem cell transplantation (HSCT) is the only curative strategy for primary myelofibrosis or post-polycythemia vera/essential thrombocythemia myelofibrosis, although novel Janus kinase (JAK) inhibitors have recently been introduced in clinical practice. However, in myelofibrosis patients undergoing reduced intensity conditioning HSCT, the engraftment failure is reported between $8 \%$ and $17 \%$ (Patriarca et al. 2008; Stewart et al. 2010; Robin et al. 2011). In addition, the presence of massive splenomegaly is considered to be a risk factor for graft failure or delayed engraftment (Stewart et al. 2010). In some patients, splenectomy is performed prior

Received July 30, 2012; accepted October 12, 2012. Published online October 27, 2012; doi: 10.1620/tjem.228.295.

Correspondence: Tatsuo Ichinohe*, M.D., Department of Hematology and Oncology, Graduate School of Medicine, Kyoto University,

54 Shogoin Kawaharacho, Sakyo-ku, Kyoto 606-8507, Japan.

e-mail: nohe@kuhp.kyoto-u.ac.jp

*Present Address: Division of Hematology, Respiratory Medicine, and Oncology, Department of Internal Medicine, Faculty of Medicine, Saga University.

e-mail: nohe@cc.saga-u.ac.jp 
to allogeneic HSCT in order to improve the likelihood of engraftment. Splenectomy before HSCT results in the faster leukocyte engraftment and the better engraftment probability, but the effect on treatment-related mortality, relapse rate, and overall survival is still debatable (Kroger et al. 2009; Bacigalupo et al. 2010; Ballen et al. 2010; Stewart et al. 2010; Robin et al. 2011). Moreover, splenectomy can cause undesired complications (Bacigalupo et al. 2010). Therefore, splenectomy prior to HSCT is not routinely recommended strategy.

Splenic irradiation is one of the methods employed for the palliative management of patients with myelofibrosis and massive splenomegaly. Splenic irradiation in the palliative setting immediately relieves the patient's symptoms, but a short duration of effectiveness and severe pancytopenia were found to be major concerns (Elliott et al. 1998; Bouabdallah et al. 2000). Because such cytopenia after irradiation can be rescued by the infusion of allogeneic stem cell grafts, we assumed that splenic irradiation as a component of a reduced-intensity conditioning regimen could favorably influence on donor cell engraftment by decreasing the size of the spleen. Here, we report two myelofibrosis patients who received splenic irradiation immediately prior to allogeneic HSCT with a reducedintensity conditioning regimen.

\section{Patient 1}

A 60-year-old woman, who had been diagnosed with essential thrombocythemia 10 years previously, presented with progressive splenomegaly, which resulted in her spleen being located $6 \mathrm{~cm}$ below the left costal margin; leukocytosis; and transfusion-dependent anemia. She was diagnosed with post-essential thrombocythemia myelofibrosis. After the written informed consent was obtained, she was treated with splenic irradiation (10 Gy in 1 Gy fraction) on days -22 through -9 , followed by reduced-intensity allogeneic HSCT according to the protocol approved by the ethics committee of Kyoto University Graduate School of Medicine. The conditioning regimen consisted of fludarabine (days -6 through -2 , total dose $125 \mathrm{mg} / \mathrm{m}^{2}$ ), oral busulfan (days -3 and -2 , total dose of $8 \mathrm{mg} / \mathrm{kg}$ ), and 2 Gy total body irradiation on day-1. Her donor was an HLA-A, -B, -C, -DRB1 allele matched sibling male donor. She was infused with a peripheral blood stem cell graft containing $2.72 \times 10^{6} \mathrm{CD} 34+$ cells $/ \mathrm{kg}$ on day 0 . The graft-versus-host disease (GVHD) prophylaxis involved the continuous injection of tacrolimus and methotrexate (Fig. 1).

During splenic irradiation, her spleen gradually reduced in size until it was located $3 \mathrm{~cm}$ below the left costal margin, and her abdominal pain was immediately relieved. Although further reduction was not evident after HSCT, improvement of splenomegaly was durable. Neutrophil engraftment, which was defined as the first consecutive days on which an absolute neutrophil count of at least $500 / \mu$ l was detected, occurred on day 13 . Prednisolone was added on day 28 because acute GVHD of the intestine was suspected. She was discharged 44 days after the transplant without any other severe complications. Acute GVHD of the skin occurred on day 71, and chronic GVHD of the liver, skin, and oral mucosa requiring additional immunosuppressive therapy occurred 5 months after the transplant. No increase in spleen size was observed until she relapsed. Unfortunately, 3 years and 2 months after the transplant her disease relapsed and progressed to acute

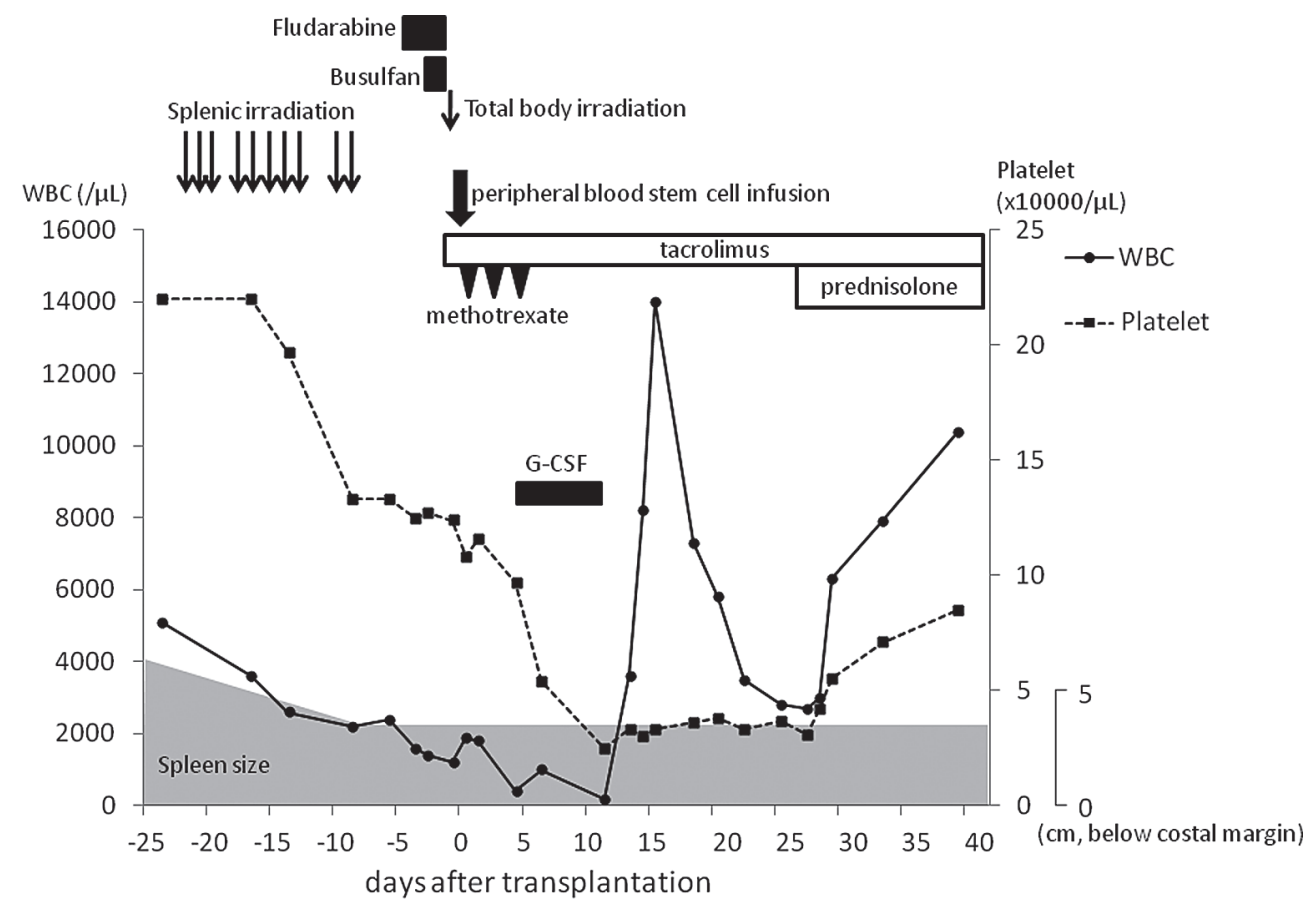

Fig. 1. Clinical course of patient 1. 


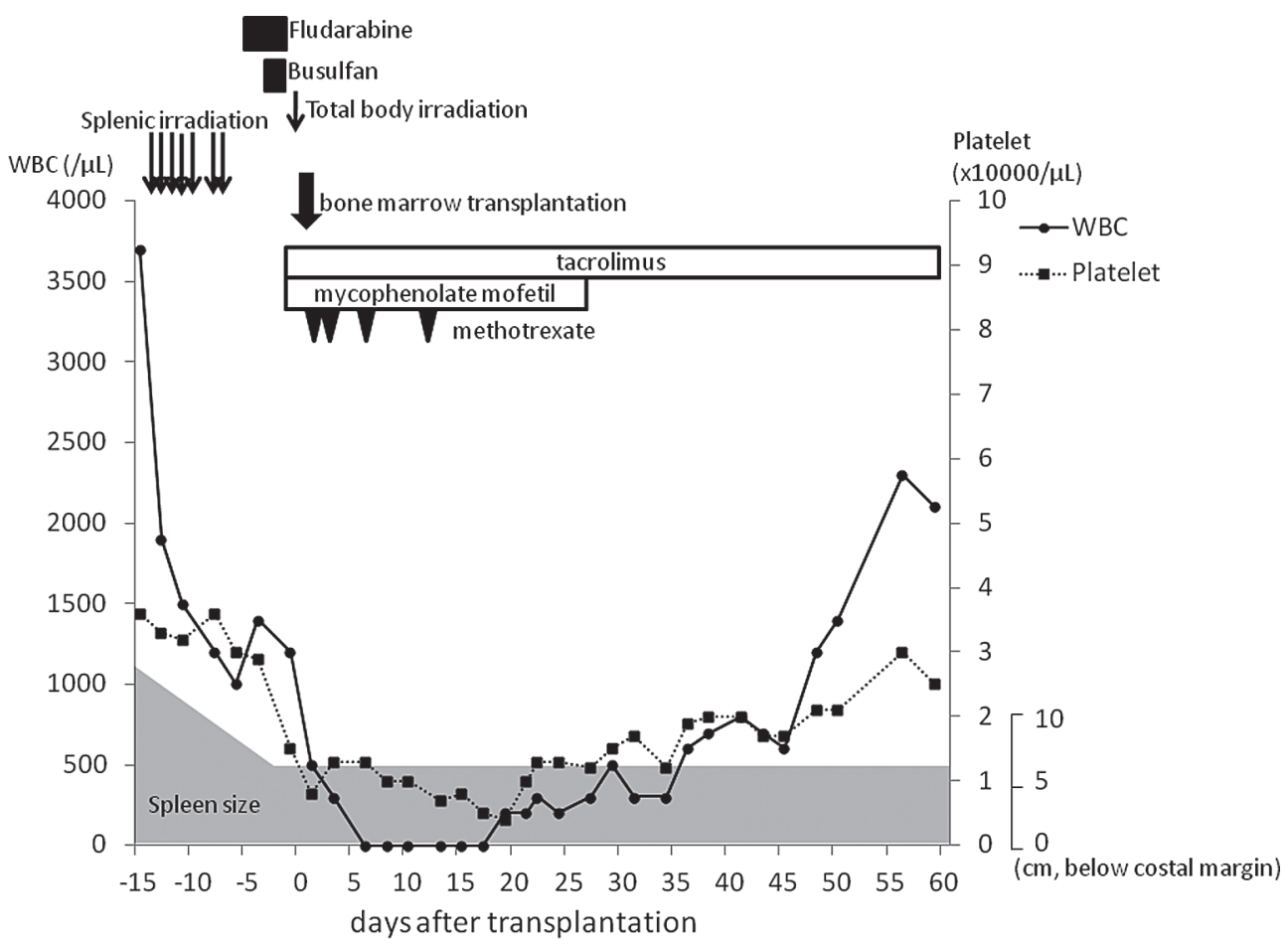

Fig. 2. Clinical course of patient 2.

myeloid leukemia. She died of the relapsed disease 5 years after the first transplant, even though a second transplant was performed.

\section{Patient 2}

A 60-year-old man suffering from progressive fatigue; abdominal pain combined with massive splenomegaly, which had reached his pelvis; transfusion-dependent anemia; thrombocytopenia; and leukoerythroblastosis was diagnosed with JAK2 V617F-associated primary myelofibrosis. After the written informed consent was obtained, he was treated with splenic irradiation (7 Gy in 1 Gy fraction) on days -15 through -7 followed by reduced-intensity allogeneic HSCT. The conditioning regimen consisted of fludarabine (total dose $125 \mathrm{mg} / \mathrm{m}^{2}$ ), intravenous busulfan (total dose $6.4 \mathrm{mg} / \mathrm{kg}$ ), and total body irradiation (2 Gy). On day 0 , the patient was infused with $2.76 \times 10^{8}$ cells $/ \mathrm{kg}$ bone marrow nucleated cells from an HLA-A, -B, -C, and DRB1 allele matched unrelated male donor. The graft-versus-host disease (GVHD) prophylaxis consisted of the continuous injection of tacrolimus, methotrexate, and mycophenolate mofetil (Fig. 2).

During the splenic irradiation, his spleen gradually decreased in size until it was located $6 \mathrm{~cm}$ below the left costal margin. No other splenic irradiation-related complications occurred except for mild nausea. Although the pace of neutrophil recovery was relatively slow without the posttransplant administration of granulocyte colony stimulating factor, the absolute neutrophil count constantly exceeded $500 / \mu 1$ after day 48 . No severe complications were observed until the patient was discharged (day 60). Acute
GVHD of the skin was observed on day 41, but improved without additional immunosuppressive therapy. Six months after the transplantation he suffered chronic GVHD of the liver and gut, but it improved after the administration of steroids. His spleen did not subsequently increase in size, and he is still alive without any sign of relapse at one year and eight months after the transplant.

\section{Discussion}

In reduced-intensity HSCT for myelofibrosis, engraftment delay or failure is the primary concern. In a large prospective study from European Group for Blood and Marrow Transplantation, engraftment failure occurs only 2 in 103 myelofibrosis patients (Kroger et al. 2009). But in this study, $11 \%$ received an additional stem cell boost after transplantation because of poor graft function. In other recent studies, engraftment failure was reported to occur in $8 \%$ to $17 \%$ patients in reduced-intensity conditioning (Patriarca et al. 2008; Stewart et al. 2010; Robin et al. 2011). The massive splenomegaly which is sometimes seen in myelofibrosis can lead to delayed engraftment and graft failure. To improve the likelihood of engraftment, splenectomy prior to HSCT is performed. Splenectomy results in the faster leukocyte engraftment (Kroger et al. 2009; Stewart et al. 2010) and the better engraftment probability (Robin et al. 2011). Robin et al. (2011) reported the improvement of overall survival by splenectomy before HSCT, but other reports did not show the favorable influence of splenectomy on non-relapse mortality and overall survival (Patriarca et al. 2008; Kroger et al. 2009; Bacigalupo et al. 2010; Ballen et al. 2010; Stewart et al. 
2010). Concerning relapse, the effect of splenectomy differs between reports. Kroger et al. (2009) reported the increase of relapse rate in splenectomized patients, but Bacigalupo et al. (2010) reported the decrease of relapse related death of splenecomized patients with large splenomegaly. Moreover, splenectomy is associated with perioperative complications, such as bleeding, infection, and thrombosis. Although Bacigalupo et al. (2010) reported that splenectomy before HSCT was not associated with increased mortality, six and five in 28 patients experienced deep vein thrombosis and febrile left pleural effusion, respectively. Among a total of 223 patients who underwent splenectomy in the palliative care setting, 68 patients suffered perioperative complications and 20 patients experienced fatal complications (Tefferi et al. 2000). Taken together, performing splenectomy prior to allogeneic HSCT for patients with massive splenomegaly is not routinely recommended. Novel JAK inhibitor therapy immediately induces reduction of splenomegaly and amelioration of constitutional symptoms (Harrison et al. 2012; Verstovsek et al. 2012). Although the effect of JAK inhibitors on transplanted hematopoietic stem cells and the withdrawal response after transplantation is not fully understood, the use of JAK inhibitors in combination with HSCT should be the area of further research.

Among chronic myeloid leukemia patients, splenic irradiation involving total doses of between $2.5 \mathrm{~Gy}$ and 10 Gy was safely performed in an attempt to enhance tumor cytoreduction, and it contributed to improvements in survival in some situations (Gratwohl et al. 1996; Jabro et al. 1999). To our knowledge, there have been no published studies investigating the role of splenic irradiation prior to allogeneic HSCT in myelofibrosis. In our two patients, splenic irradiation immediately relieved the patients' symptoms and successfully reduced the size of their spleens before the allograft infusion. In Patient 2, although neutrophil engraftment was delayed, severe leukocytopenia less than $200 / \mu 1$ was not observed after day 19 and eventually successful engraftment was confirmed. In both patients, no severe splenic irradiation-related complications were observed.

In conclusion, our present experience suggests that splenic irradiation prior to allogeneic HSCT is feasible and might facilitate engraftment without having adverse effects on the outcome of allogeneic HSCT for selected patients with primary or post-polycythemia vera/essential thrombocythemia myelofibrosis. Future studies are warranted to evaluate the role of splenic irradiation as a component of a reduced-intensity conditioning regimen in allogeneic HSCT for myelofibrosis with massive splenomegaly.

\section{References}

Bacigalupo, A., Soraru, M., Dominietto, A., Pozzi, S., Geroldi, S., Van Lint, M.T., Ibatici, A., Raiola, A.M., Frassoni, F., De Stefano, F., Verdiani, S., Casarino, L. \& Barosi, G. (2010) Allogeneic hemopoietic SCT for patients with primary myelo- fibrosis: a predictive transplant score based on transfusion requirement, spleen size and donor type. Bone Marrow Transplant., 45, 458-463.

Ballen, K.K., Shrestha, S., Sobocinski, K.A., Zhang, M.J., Bashey, A., Bolwell, B.J., Cervantes, F., Devine, S.M., Gale, R.P., Gupta, V., Hahn, T.E., Hogan, W.J., Kroger, N., Litzow, M.R., Marks, D.I., Maziarz, R.T., McCarthy, P.L., Schiller, G., Schouten, H.C., Roy, V., Wiernik, P.H., Horowitz, M.M., Giralt, S.A. \& Arora, M. (2010) Outcome of transplantation for myelofibrosis. Biol. Blood Marrow Transplant., 16, 358-367.

Bouabdallah, R., Coso, D., Gonzague-Casabianca, L., Alzieu, C., Resbeut, M. \& Gastaut, J.A. (2000) Safety and efficacy of splenic irradiation in the treatment of patients with idiopathic myelofibrosis: a report on 15 patients. Leuk. Res., 24, 491-495.

Cervantes, F., Dupriez, B., Pereira, A., Passamonti, F., Reilly, J.T., Morra, E., Vannucchi, A.M., Mesa, R.A., Demory, J.L., Barosi, G., Rumi, E. \& Tefferi, A. (2009) New prognostic scoring system for primary myelofibrosis based on a study of the International Working Group for Myelofibrosis Research and Treatment. Blood, 113, 2895-2901.

Elliott, M.A., Chen, M.G., Silverstein, M.N. \& Tefferi, A. (1998) Splenic irradiation for symptomatic splenomegaly associated with myelofibrosis with myeloid metaplasia. Br. J. Haematol., 103, 505-511.

Gratwohl, A., Hermans, J., van Biezen, A., Arcese, W., Debusscher, L., Ernst, P., Ferrant, A., Frassoni, F., Gahrton, G., Iriondo, A., Kolb, H.J., Link, H., Niederwieser, D., Ruutu, T., Siegert, W. \& Zwaan, F.E. (1996) Splenic irradiation before bone marrow transplantation for chronic myeloid leukaemia. Chronic Leukaemia Working Party of the European Group for Blood and Marrow Transplantation (EBMT). Br. J. Haematol., 95, 494-500.

Harrison, C., Kiladjian, J.J., Al-Ali, H.K., Gisslinger, H., Waltzman, R., Stalbovskaya, V., McQuitty, M., Hunter, D.S., Levy, R., Knoops, L., Cervantes, F., Vannucchi, A.M., Barbui, T. \& Barosi, G. (2012) JAK inhibition with ruxolitinib versus best available therapy for myelofibrosis. N. Engl. J. Med., 366, 787-798.

Jabro, G., Koc, Y., Boyle, T., Schenkein, D.P., Ravalese, J., Wazer, D. \& Miller, K.B. (1999) Role of splenic irradiation in patients with chronic myeloid leukemia undergoing allogeneic bone marrow transplantation. Biol. Blood Marrow Transplant., 5, 173-179.

Kröger, N., Holler, E., Kobbe, G., Bornhauser, M., Schwerdtfeger, R., Baurmann, H., Nagler, A., Bethge, W., Stelljes, M., Uharek, L., Wandt, H., Burchert, A., Corradini, P., Schubert, J., Kaufmann, M., Dreger, P., Wulf, G.G., Einsele, H., Zabelina, T., Kvasnicka, H.M., Thiele, J., Brand, R., Zander, A.R., Niederwieser, D. \& de Witte, T.M. (2009) Allogeneic stem cell transplantation after reduced-intensity conditioning in patients with myelofibrosis: a prospective, multicenter study of the Chronic Leukemia Working Party of the European Group for Blood and Marrow Transplantation. Blood, 114, 5264-5270.

Patriarca, F., Bacigalupo, A., Sperotto, A., Isola, M., Soldano, F., Bruno, B., van Lint, M.T., Iori, A.P., Santarone, S., Porretto, F., Pioltelli, P., Visani, G., Iacopino, P., Fanin, R. \& Bosi, A. (2008) Allogeneic hematopoietic stem cell transplantation in myelofibrosis: the 20-year experience of the Gruppo Italiano Trapianto di Midollo Osseo (GITMO). Haematologica, 93, 1514-1522.

Robin, M., Tabrizi, R., Mohty, M., Furst, S., Michallet, M., Bay, J.O., Cahn, J.Y., De Coninck, E., Dhedin, N., Bernard, M., Rio, B., Buzyn, A., Huynh, A., Bilger, K., Bordigoni, P., Contentin, N., Porcher, R., Socie, G. \& Milpied, N. (2011) Allogeneic haematopoietic stem cell transplantation for myelofibrosis: a report of the Societe Francaise de Greffe de 
Moelle et de Therapie Cellulaire (SFGM-TC). Br. J. Haematol., 152, 331-339.

Stewart, W.A., Pearce, R., Kirkland, K.E., Bloor, A., Thomson, K., Apperley, J., McQuaker, G., Marks, D.I., Craddock, C., McCann, S., Russell, N., Cook, G. \& Kottaridis, P.D. (2010) The role of allogeneic SCT in primary myelofibrosis: a British Society for Blood and Marrow Transplantation study. Bone Marrow Transplant., 45, 1587-1593.

Tefferi, A., Mesa, R.A., Nagorney, D.M., Schroeder, G. \& Silverstein, M.N. (2000) Splenectomy in myelofibrosis with myeloid metaplasia: a single-institution experience with 223 patients. Blood, 95, 2226-2233.

Verstovsek, S., Mesa, R.A., Gotlib, J., Levy, R.S., Gupta, V., DiPersio, J.F., Catalano, J.V., Deininger, M., Miller, C., Silver, R.T., Talpaz, M., Winton, E.F., Harvey, J.H. Jr., Arcasoy, M.O., Hexner, E., Lyons, R.M., Paquette, R., Raza, A., Vaddi, K., Erickson-Viitanen, S., Koumenis, I.L., Sun, W., Sandor, V. \& Kantarjian, H.M. (2012) A double-blind, placebo-controlled trial of ruxolitinib for myelofibrosis. N. Engl. J. Med., 366, 799-807. 